\title{
Stabilization with Prescribed Instant for High-Order Integrator Systems
}

\author{
Jiyuan Kuang, Jianxing Liu, Senior Member, IEEE, Yabin Gao, Member, IEEE, Chih-Chiang Chen, Member, \\ IEEE, Xiaoju Zhang, Guanghui Sun, Senior Member, IEEE,
}

\begin{abstract}
This paper develops a new controller design approach to stabilize system states $x$ onto the equilibrium at an arbitrarily selected time instant $t_{f}$ irrespective of initial system states $x_{0}$ and parameters. Therefore, the actual convergence time is independent with $x_{0}$, and $\dot{x} \neq 0$ until $t \geq t_{f}$, which is different with traditional fixed, predefined, and prescribed time stability. In this work, a series of potential practicable time-varying feedback functions are explored, by which a novel controller is designed for high-order integrator systems. The proposed control is bounded and can gradually go to zero at an arbitrarily selected time instant, at which the system states reach zero simultaneously. This special stability of the controlled system is analyzed by reduction to absurdity rather than the Lyapunov method. The effectiveness of the proposed results is illustrated by numerical simulations.
\end{abstract}

Index Terms-Finite-time control, Prescribed-time control, Time-varying feedback, Reduction to absurdity

\section{INTRODUCTION}

A $\mathrm{S}$ is well-known, the central philosophy of finite-time stabilization (FNTS) is the guarantee of the state convergence over a finite time interval while providing an estimation of the convergence time (i.e., the settling time) depending on initial states and control parameters [1], [2]. For autonomous systems, the finite-time stability analysis was formally presented in [3] in which a Lyapunov-based inequality $\dot{V}(x) \leq$ $-a V^{b}(x)$ with $b \in(0,1)$ and $a>0$ was developed. The real convergence time $T\left(x_{0}\right)$ satisfies $T\left(x_{0}\right) \leq \frac{V\left(x_{0}\right)^{1-b}}{a(1-b)}$, and there is no uniform bounds for $T\left(x_{0}\right), \forall x_{0} \in \mathbb{R}^{n}$. Many variants around this Lyapunov inequality were further proposed, for example, the homogeneous approach [4], the implicit Lyapunov function approach [5], the adaptive control [6]. These years, the FNTS method was further developed as three branches: the fixed-time stabilization (FTS), the predefined-time stabilization (PDTS), and the prescribed-time stabilization (PSTS). These control methods are classified into two categories, one is the FTS and the PDTS, the other is the PSTS.

The FTS and the PDTS get finite-time stability by utilizing the power of some special functions of system states. the

This work was supported in part by the National Key R\&D Program of China (2019YFB1312001), the National Natural Science Foundation of China (62022030 and 62033005), and the state grid heilongjiang electric power company limited funded project (No. 522417190057). Corresponding author: Jianxing Liu (Email: jx.liu@hit.edu.cn)

J. Kuang, J. Liu, Y. Gao, and G. Sun are with the School of Astronautics, Harbin Institute of Technology, Harbin 150001, China.

C.-C. Chen is with the Department of Systems and Naval Mechatronic Engineering, National Cheng Kung University, Tainan 70101, Taiwan

X. Zhang is with the School of Mathematics, Harbin Institute of Technology, Harbin 150001, P. R. China
FTS ensures the convergence time $T\left(x_{0}\right)$ to be bounded by a constant $T_{\max }$, which is independent of the initial conditions $x_{0}$, in another word, $T\left(x_{0}\right) \leq T_{\max }, \forall x_{0} \in \mathbb{R}^{n}$ [7], [8]. The constant $T_{\max }$ is determined by some controller parameters, so one should calculate the parameters according to an assigned $T_{\max }$. As two important applications, fixed-time differentiator [9] and fixed-time observer [10] were then proposed. Moreover, the robust FTS of MIMO systems under matched and unmatched disturbances were also studied [11]. However, the actual convergence time may be far smaller than the designed $T_{\max }$ [12]. Hence, it is logical to pursue less conservativeness in the FTS. To this end, the notion of the PDTS was proposed as a result of $\sup _{\forall x_{0} \in \mathbb{R}^{n}} T\left(x_{0}\right)=T_{f}$ [13], [14]. Another improvement of the PDTS among the FTS is that the assigned time $T_{f}$ is a constant parameter explicit in the controller. This feature is quite similar to the PSTS. One defect of the traditional predefined-time controller is that the initial control grows exponentially with $x_{0}$. This weakness was overcame by the construction of a series of new controllers with polynomial terms instead of exponential ones [15].

The idea of the PSTS is inspired by some time-varying feedback functions that seem to go to infinity towards the terminal time $T_{f}$ [16]. This method ensures $T\left(x_{0}\right) \leq T_{f}, \forall x_{0} \in \mathbb{R}^{n}$, where $T_{f}$ is a constant parameter can be arbitrarily assigned and is explicit in the controller [17], [18]. The PSTS was combined with parametric Lyapunov equations and then got a uniformly bound of the control variable [19]. The PSTS was also appropriately recast within the framework of time-varying homogeneity [20]. Moreover, the PSTS with uncertainties was studied for nonlinear systems [21], [22]. Compared with the PDTS, the assigned time $T_{f}$ of the PSTS is more conservative. It is noted that the singularity or discontinuity of prescribedtime control may appear when $t$ tends to $t_{0}+T_{f}$, as there is usually a term $\frac{1}{t_{0}+T_{f}-t}$ in time-varying feedback functions. Hence, such controllers on PSTS should be carefully designed to ensure their boundedness [23].

Currently, the methods to achieve the FNTS introduced above have been combined and learned from each other. Some interesting achievements and applications are listed below. Notably, by designing autonomous stabilizing controllers with some added time-varying gains, the work in [24] derived a predefined upper bound of the settling time and provided sufficient conditions such that the time-varying gains remain bounded. To mention a few, Michiels et al. generalized the fixed-time control to linear systems with input delays by using the so-called act-and-wait control strategy [25]. Hu et al. improved the FTS to deal with the problem of synchronization for 
discontinuous dynamic networks [26]. Zhang et al. proposed a novel bipartite fixed-time observer to address the bipartite fixed-time output consensus problem of heterogeneous linear multiagent systems [27]. Kunal et al. proposed a fixed-time stable gradient-flow scheme to yield convergence to an optimal point of a convex optimization problem within a fixed time [28]. Mechali et al. proposed an observer-based fixed-time continuous nonsingular terminal sliding mode control method to solve the problem of robust trajectory tracking for a quadrotor aircraft under disturbances [29].

To sum up, the abovementioned methods more or less have some conservativeness on the real convergence time $T\left(x_{0}\right)$, i.e., $T\left(x_{0}\right) \leq T_{f}, \forall x_{0} \in \mathbb{R}^{n}$ and $T\left(x_{0}\right) \leq T_{\max }, \forall x_{0} \in \mathbb{R}^{n}$. This paper aims to design a controller based on the $n$-order chain of integrators to achieve $T\left(x_{0}\right)=T_{f}, \forall x_{0} \in \mathbb{R}^{n}$. Firstly, the $n$-order chain of integrators is transformed to a set of differential equations containing special time-varing functions. Secondly, the transformed system is proved to converge to zero at a selected time instant. Then, the convergence of the original system and the transformed system are proved to be equivalent. This solution is also inspired by time-varying feedback functions, and somewhat belongs to the PSTS. Moreover, the convergence to zero happening at a selected time instant is guaranteed. For these reasons, the proposed stabilization method of this paper is called the prescribedinstant stabilization (PSIS ${ }^{1}$ ) in the following context. Our main contribution can be summarized as follows:

a) Several new time-varying feedback functions are proposed.

b) A recursive controller based on the $n$-order chain of integrators is proposed by applying the time-varying feedback functions.

c) The prescribed-instant stability is proved by reduction to absurdity, which is rarely utilized in the stability analysis.

The rest of the paper is structured as follows: Section II provides some basic knowledge and followed the problem statement. Section III gives some new time-varying feedback functions, based on which the general form of the controller is presented. Then reduction to absurdity is utilized in the analysis of the PSIS. In Section IV, simulations are provided to validate the proposed results. Section V concludes the paper.

\section{PRELIMINARIES AND PROBLEM STATEMENT}

\section{A. Preliminaries}

Consider the following autonomous system:

$$
\dot{x}=f(x, \eta), x\left(t_{0}\right)=x_{0},
$$

where $x \in \mathbb{R}^{n}$ is the system state, and the vector $\eta \in \mathbb{R}^{l}$ stands for the tunable parameters of (1). The function $f$ : $\mathbb{R}_{\geq 0} \times \mathbb{R}^{n} \rightarrow \mathbb{R}^{n}$ may be discontinuous, and such solutions of (1) exist and are unique in the sense of Filippov [30]. Thus, $\Phi\left(t, x_{0}\right)$ denotes the solution of (1) starting from $x_{0} \in \mathbb{R}^{n}$ at $t=0$. Moreover, the origin $x=0$ is the unique equilibrium point of (1).

\footnotetext{
${ }^{1}$ The PSIS will be the abbreviation of "prescribed-instant stable", "prescribed-instant stability" and "prescribed-instant stabilization" without distinction in the context.
}

As stated in [31], the parameter-dependent system (1) is equivalent to the controlled system

$$
\dot{x}=g(x, u),
$$

where $g: \mathbb{R}^{n} \times \mathbb{R}^{m} \rightarrow \mathbb{R}^{n}$, the control $u \in \mathbb{R}^{m}$ is a feedback function of $x$ with tunable parameters $\eta$, i.e., $u=w(x, \eta)$, with $w: \mathbb{R}^{n} \rightarrow \mathbb{R}^{m}$. Substituting $u=w(x, \eta)$ in (2) eliminates $u$ and yields $f(x, \eta):=g(x, w(x, \eta))$. All the notions defined and treated hereafter are global, so we will omit to indicate it.

Definition 1: The origin of the system (1) is said to be

(1) finite-time stable (FNTS) [1], if it is Lyapunov stable and for any $x_{0} \in \mathbb{R}^{n}$ there exists $\mathcal{T}>0$ such that $\Phi\left(t, x_{0}\right)=$ $0, \forall t \geq t_{0}+\mathcal{T}$. The settling-time function of (1) is defined as, $T\left(x_{0}\right)=\inf \left\{\mathcal{T} \geq 0: \Phi\left(t, x_{0}\right)=0, \forall t \geq t_{0}+\mathcal{T}\right\}$.

(2) fixed-time stable (FTS) [8], if it is finite-time stable and the settling-time function is bounded, i.e., $\exists 0<T_{\max }<\infty$ such that $T\left(x_{0}\right) \leq T_{\max }, \forall x_{0} \in \mathbb{R}^{n}$.

Remark 1: It is important to highlight that $T_{\max }$ is usually a function of $\eta$, and $T_{\max }$ is not explicit in the control $u$. On the other hand, $T_{\max }$ can be chosen larger than the calculated result from $\eta$. For instance, if the settling time function is bounded by $T_{\mathrm{m}}$, it is also bounded by $\lambda T_{\mathrm{m}}, \forall \lambda \geq 1$. How to find the smallest $T_{\max }$ motivates the following definition.

Definition 2: [12] The origin of the system (1) is said to be predefined-time stable (PDTS) if it is fixed-time stable, and for any positive nunmber $T_{f}$, there exists some $\eta \in \mathbb{R}^{l}$ such that either of the following can be established,

(a) $T\left(x_{0}\right) \leq T_{f}, \forall x_{0} \in \mathbb{R}^{n}$ (weak-predefined time)

(b) $\sup _{\forall x_{0} \in \mathbb{R}^{n}} T\left(x_{0}\right)=T_{f}$ (strong-predefined time)

Remark 2: In a strict sense, the time $T_{f}$ can be considered as the true fixed-time in which the system (1) is stabilized. Moreover, $T_{f}$ used in PDTS is usually a single constant parameter explicit in the controller, i.e., $u=w\left(x, \eta, T_{f}\right)$.

The FNTS, the FTS, and the PDTS are realized by the control $u=w(x, \eta)$ independent with time $t$. Now, let us consider a time-varing control $u=w(x, t, \eta)$. Then, substituting it into (2) yields a nonautonomous system:

$$
\dot{x}=f(x, t, \eta):=g(x, w(x, t, \eta)), x\left(t_{0}\right)=x_{0} .
$$

Definition 3: [18], [23] The origin of the system (3) is said to be prescribed-time stable (PSTS) if it is finite-time stable, and for any positive nunmber $T_{f}$, there exists some $\eta \in \mathbb{R}^{l}$ such that $T\left(x_{0}\right) \leq T_{f}, \forall x_{0} \in \mathbb{R}^{n}$.

Remark 3: $T_{f}$ in PSTS is usually a constant parameter (independedt with other parameters) explicit in the control, i.e., $u=w\left(x, t, \eta, T_{f}\right)$. In a strict sense, existing proof of PSTS only demonstrated that $T\left(x_{0}\right) \leq T_{f}$, so there may exist a positive number $T_{c}<T_{f}$ such that $x=0, \dot{x}=0, \forall t \geq t_{0}+T_{c}$. To eliminate this conservativeness, we propose the following definition.

Definition 4: Consider that the origin of the system (3) is prescribed-time stable with $T_{f}>0$. Then, the origin of the system (1) is prescribed-instant stable (PSIS) if $T\left(x_{0}\right)=T_{f}$.

Remark 4: $T_{f}=t_{f}-t_{0}$ is the assigned convergence time, and $t_{f}$ is the assigned instant when the system states convergent to zero. $T\left(x_{0}\right)=t_{a}-t_{0}$ is the actual convergence time or settling time, and $t_{a}$ is the actual instant when the system states 
convergent to zero. It is noted that the proposed prescribedinstant stability in this paper means the extraordinary accuracy on time, i.e., the true convergence time exactly equal to the assigned time. Therefore, $T\left(x_{0}\right)$ is actually independent with $x_{0}$, and $\dot{x} \neq 0$ until $t \geq t_{f}$. It is obviously different with the FTS, the PDTS, and the PSTS.

\section{B. Comparison of Control Structures}

Consider the following single-integrator system:

$$
\dot{x}=u, x\left(t_{0}\right)=x_{0}>0, t_{0}=0 .
$$

1) Traditional finite-time control [3]:

$$
\begin{aligned}
& u_{\mathrm{FNTS}}=-\eta_{1} x^{1-\frac{1}{\eta_{2}}}, \eta_{1}>0, \eta_{2} \in(1, \infty), \\
& T\left(x_{0}\right)=\frac{\eta_{2} x^{\frac{1}{\eta_{2}}}}{\eta_{1}}
\end{aligned}
$$

2) Fixed-time control [8]:

$$
\begin{aligned}
& u_{\mathrm{FTS}}=-x^{\eta_{3}}-x^{\eta_{4}}, \eta_{3} \in(0,1), \eta_{4} \in(1, \infty), \\
& T\left(x_{0}\right) \leq T_{\max }=\frac{1}{\left(1-\eta_{3}\right)}+\frac{1}{\left(\eta_{4}-1\right)} .
\end{aligned}
$$

3) Predefined-time control [12]:

$$
\begin{aligned}
& u_{\text {PDTS }}=-\frac{\eta_{5}}{T_{f}} \mathrm{e}^{x^{\frac{1}{\eta_{5}}}} x^{1-\frac{1}{\eta_{5}}}, \eta_{5} \in(1, \infty), \\
& \sup _{\forall x_{0} \in \mathbb{R}} T\left(x_{0}\right)=T_{f} .
\end{aligned}
$$

4) Prescribed-time control [18]:

$$
\begin{aligned}
& u_{\mathrm{PSTS}}= \begin{cases}-\frac{\eta_{6} x}{T_{f}-t}, \eta_{6} \in(1, \infty), & 0 \leq t<T_{f}, \\
0, & T_{f} \leq t .\end{cases} \\
& T\left(x_{0}\right) \leq T_{f} .
\end{aligned}
$$

Remark 5: It is interesting to notice that the solution of system in (4) under the control $u_{\text {PSTS }}$ is $x=\left(T_{f}-t\right)^{\eta_{6}}$, which is also the solution of

$$
\dot{x}=u=-\frac{\eta_{6}}{T_{f}} x_{0}^{\frac{1}{\eta_{6}}} x^{1-\frac{1}{\eta_{6}}} .
$$

One can see that the prescribed-time control is very similar to predefined-time control. Moreover, taking $\frac{\eta_{6}}{T_{f}} x_{0}^{\frac{1}{\eta_{6}}}$ as $\eta_{1}$ shows the unification of the PSTS and the FNTS.

\section{Problem Statement}

It is known for all that a SISO nonlinear system with a relative degree $n$ can usually be transformed to the $n$-order chain of integrators by utilizing input-output linearization method (Section 2, Chapter 13 of [32]). A controllable MIMO linear system can also be transformed to the controller canonical form, which can be seen as the combination of several chains of integrators. Hence, let us consider the following $n$-order chain of integrators:

$$
\left\{\begin{array}{l}
\dot{x}_{i}=x_{i+1}, i=1, \ldots, n-1, \\
\dot{x}_{n}=u
\end{array}\right.
$$

For a designed time varying control $u(x, t, \eta)$, the system (5) belongs to the form in (1). Our goal is to
1) design a continuous control $u(x, t, \eta)$ goes to zero at a selected instant $t_{f}$, and

2) ensure that the equilibrium of the system in (5) is PSIS by exploring new techniques of stability analysis.

\section{MAIN RESUltS}

In this section, the method to design the PSIS controller for the system (5) is presented. Before that, some new timevarying feedback functions, the raw material of the presented controller, are introduced.

\section{A. Time-varying Feedback Functions}

Definition 5: Consider a differentiable function $\phi(s) \in$ $\kappa, \kappa_{\infty}$ (the class $\kappa$ or $\kappa_{\infty}$ function [32]), and $s=\operatorname{arc}(\phi(s))$ (the existence of inverse function is guaranteed by the monotonicity of $\phi$ ). Suppose that $\phi$ satisfy

- $\phi(s) \sim s$ as $s \rightarrow 0$, i.e. $\lim _{s \rightarrow 0} \frac{\phi(s)}{s}=1$,

- $\phi^{\prime}(s)=\beta(\phi(s))$.

Let $s(t)=\left(t_{f}-t\right)^{\eta}, x(t)=\phi(s(t))=\phi\left(\left(t_{f}-t\right)^{\eta}\right), t \in$ $\left[0, t_{f}\right], \eta>1$. We have

$\frac{\mathrm{d} x}{\mathrm{~d} t}=\frac{\mathrm{d} \phi}{\mathrm{d} s} \frac{\mathrm{d} s}{\mathrm{~d} t}=-\frac{\eta \beta(\phi(s)) \operatorname{arc}(\phi(s))}{t_{f}-t}=-\frac{\zeta(x)}{t_{f}-t}=-\psi(x, t)$.

Then, $\phi\left(\left(t_{f}-t\right)^{\eta}\right)$ is defined as a reference convergence function $(\mathrm{RCF})$, while $\psi(x, t)$ is defined as a reference convergence differential function (RCDF).

Remark 6: The defined RCDFs above are exactly our desired time-varying feedback functions. According to Definition 5, $\phi$ monotonously goes to zero as $s$ goes to zero, thus $\psi(\phi, t)$ and $\zeta(\phi)$ have the same sign with $\phi$ and will not be zero unless $\phi$ is zero. These functions are also expected to have some important merits. For example, $\psi(\phi, t)$ should be designed to be a symmetrical function (same properties for positive and negative $\phi$ ). It is noted that $\lim _{t \rightarrow t_{f}} \phi^{\prime}\left(\left(t_{f}-t\right)^{\eta}\right)=$ $\lim _{t \rightarrow t_{f}} \frac{\phi\left(\left(t_{f}-t\right)^{\eta}\right)-0}{t-t_{f}} \sim\left(t_{f}-t\right)^{\eta-1}$, for $\eta>1$. For the first-order system $\dot{x}=u$, if $u$ is designed as $-\psi(x, t)$, then both $u(t)$ and $x(t)$ will converge to zero at $t=t_{f}$. Let us see some examples on Definition 5.

Example 1: Notice that $x(t)=\tan \left(\left(t_{f}-t\right)^{\eta}\right)$ (an RCF) will converge to zero at $t_{f}$, we obtain the following RCDF:

$$
\dot{x}=\frac{-\eta}{\cos ^{2}\left(\left(t_{f}-t\right)^{\eta}\right)} \frac{\left(t_{f}-t\right)^{\eta}}{t_{f}-t}=\frac{-\eta\left(x^{2}+1\right) \arctan (x)}{t_{f}-t} .
$$

One can also design analogous RCDFs according to the thought obtaining the RCDF in (6), e.g.

$$
\left\{\begin{array}{l}
x=\operatorname{sh}\left(\left(t_{f}-t\right)^{\eta}\right)=\frac{\mathrm{e}^{\left(t_{f}-t\right)^{\eta}}-\mathrm{e}^{-\left(t_{f}-t\right)^{\eta}}}{2}, \\
\dot{x}=-\frac{\eta \sqrt{1+x^{2}} \ln \left(x+\sqrt{1+x^{2}}\right)}{t_{f}-t}
\end{array}\right.
$$

In this paper, the following three typical RCFs $(\phi)$ are discussed in detail,

$$
\begin{aligned}
& x_{1}=\tan \left(t_{f}-t\right)^{\eta}, \\
& x_{2}=\left(t_{f}-t\right)^{\eta}, \\
& x_{3}=\ln \left(1+\left(t_{f}-t\right)^{\eta}\right) .
\end{aligned}
$$




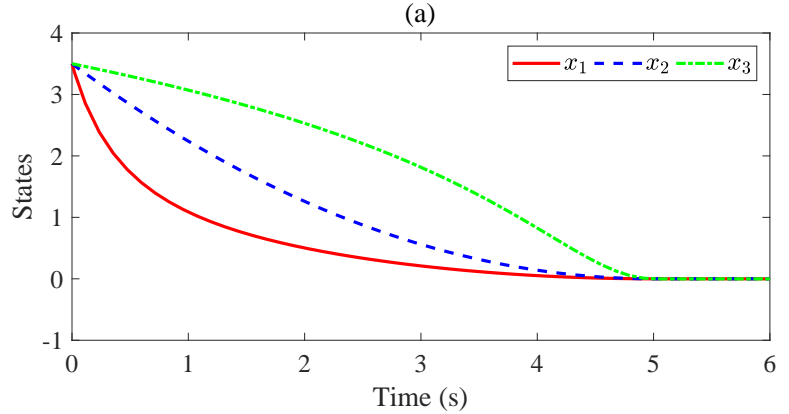

(b)

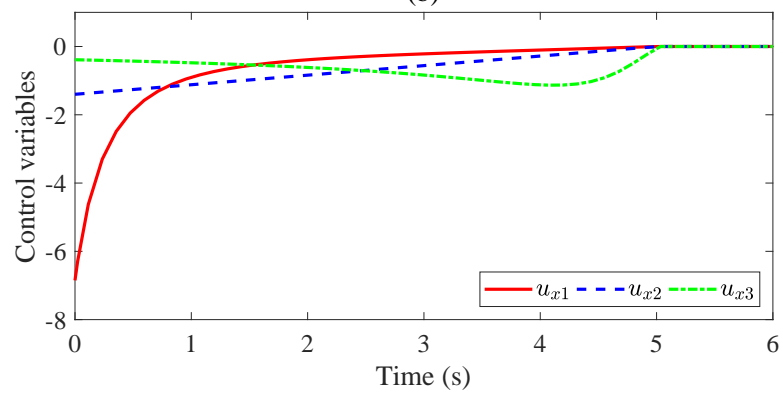

Fig. 1. States and control variables of the three typical RCDFs of (7)-(9).

The corresponding $\mathrm{RCDFs}(\psi)$ are:

$$
\begin{aligned}
& \dot{x}_{1}=u_{x 1}=-\frac{\eta\left(x_{1}^{2}+1\right) \arctan \left(x_{1}\right)}{t_{f}-t}, \\
& \dot{x}_{2}=u_{x 2}=-\frac{\eta x_{2}}{t_{f}-t}, \\
& \dot{x}_{3}=u_{x 3}=-\frac{\eta\left(1-\mathrm{e}^{-\left|x_{3}\right|}\right)}{t_{f}-t} \operatorname{sign}\left(x_{3}\right) .
\end{aligned}
$$

It is interesting to notice that the control in (8) is exactly that of PSTS with single-integrator system. However, the control for high-order integrator systems will be different. Fig. 1 shows the convergence of the states to the origin and the control variables corresponding to the RCDFs in (7)-(9), where $t_{f}=$ $5, x(0)=3.5$, and $\eta=2$.

\section{B. Controller Design}

Consider the system (5). The proposed RCDFs such as in (7)-(9) are utilized as time-varying feedback functions. The controller is demonstrated by a recursive form. The initial condition is chosen as $x_{1, d}=0$ and $x_{2, d}=-\psi_{1}$. The recurrence relation $(i \geq 2)$ is

$$
x_{i+1, d}=\dot{x}_{i, d}-\sigma_{i}-\psi_{i},
$$

where $\sigma_{i}=x_{i}-x_{i, d}$, and $\psi_{i}$ belongs to the RCDFs. To avoid divergence to infinity or discontinuity of the control law at $t=t_{f}$, we select each parameter $\eta_{i}$ of function $\psi_{i}$ as $\eta_{i}>$ $n+1-i, i=1,2, \ldots, n$. The proposed controller for the system (5) is:

$$
u_{n}= \begin{cases}x_{n+1, d}, & t_{0} \leq t<t_{f}, \\ 0, & t_{f} \leq t,\end{cases}
$$

where $x_{n+1, d}$ is obtained from (10). On the other hand, one can obtain that

$$
\dot{\sigma}_{i}=\dot{x}_{i}-\dot{x}_{i, d}=x_{i+1}-x_{i+1, d}-\sigma_{i}-\psi_{i}
$$

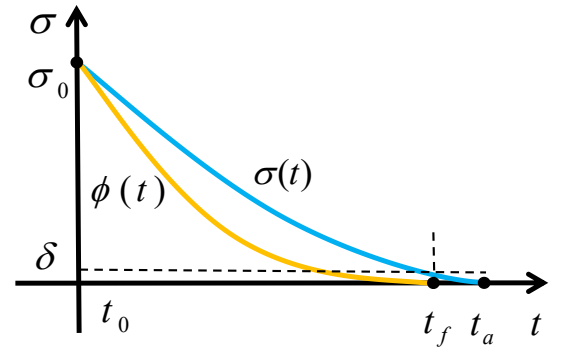

Fig. 2. The sketch map of Claim 1.

$$
\begin{aligned}
& =\sigma_{i+1}-\sigma_{i}-\psi_{i}, \\
\dot{\sigma}_{n} & =\dot{x}_{n}-\dot{x}_{n, d}=u-\dot{x}_{n, d} \\
& =-\sigma_{n}-\psi_{n} .
\end{aligned}
$$

Hence, the system (5) is transformed to:

$$
\left\{\begin{array}{l}
\dot{\sigma}_{1}=\sigma_{2}-\psi_{1}, \\
\dot{\sigma}_{i}=\sigma_{i+1}-\sigma_{i}-\psi_{i}, i=2, \ldots, n-1, \\
\dot{\sigma}_{n}=-\sigma_{n}-\psi_{n} .
\end{array}\right.
$$

It is noted that the dynamics of $\sigma_{i}$ is only affected by $\psi_{i}, \sigma_{i+1}$ and(or) itself. This is very important for our further proof of the PSIS.

\section{Prescribed-instant Stability (PSIS)}

In this subsection, we will directly study the character of the differential equations in the system (12), and illustrate the PSIS of the system (5) under the control in (11). Several claims and lemmas are introduced for developing our main results.

Claim 1: Consider the following differential equation,

$$
\dot{\sigma}=\sigma-\psi(\sigma, t)
$$

where $\psi$ is an RCDF. Then, $\sigma$ reaches zero at $t=t_{f}$.

Proof: We consider a positive initial value $\sigma\left(t_{0}\right)$ for the symmetry of the equation in (13). According to the comparison Lemma [32], $\sigma$ is lower bounded by $\phi(t)$ corresponding to the selected $\psi$ and $t_{a} \geq t_{f}$, as the illustration in Fig. 2. Reduction to absurdity is utilized as follows. We assume $t_{a}>t_{f}$, i.e., $\sigma\left(t_{f}\right)=\delta>0$.

Utilizing the mean value theorem of integrals, we have,

$$
\begin{aligned}
\sigma\left(t_{f}\right) & =\delta+\int_{t_{0}}^{t_{f}} \sigma(t) \mathrm{d} t-\int_{t_{0}}^{t_{f}} \frac{\zeta(\sigma)}{t_{f}-t} \mathrm{~d} t \\
& =k-\int_{t_{0}}^{t_{f}} \frac{\zeta(\sigma)}{t_{f}-t} \mathrm{~d} t
\end{aligned}
$$

where $k$ is a constant calculated from the first two terms and $\frac{\zeta(\sigma)}{t_{f}-t}$ is $\psi$. According to Definition 5 and Remark $6, \delta \leq$ $\sigma(t) \leq \sigma_{0}$ leads to $m \leq \zeta(\sigma) \leq M$, where $m$ and $M$ are some positive constants. We have

$$
\int_{t_{0}}^{t_{f}} \frac{m}{t_{f}-t} \mathrm{~d} t \leq \int_{t_{0}}^{t_{f}} \frac{\zeta(\sigma)}{t_{f}-t} \mathrm{~d} t \leq \int_{t_{0}}^{t_{f}} \frac{M}{t_{f}-t} \mathrm{~d} t .
$$

Let $\tau=t_{f}-t$. Then, we obtain that

$$
\int_{t_{0}}^{t_{f}} \frac{m}{t_{f}-t} \mathrm{~d} t=\int_{0}^{t_{f}-t_{0}} \frac{m}{\tau} \mathrm{d} \tau=+\infty,
$$




$$
\int_{t_{0}}^{t_{f}} \frac{M}{t_{f}-t} \mathrm{~d} t=\int_{0}^{t_{f}-t_{0}} \frac{M}{\tau} \mathrm{d} \tau=+\infty
$$

Therefore, by the Squeeze Theorem, we have $\int_{t_{0}}^{t_{f}} \frac{\zeta(\sigma)}{t_{f}-t} \mathrm{~d} t=$ $+\infty$, which leads to $\sigma\left(t_{f}\right)=-\infty$. This is in conflict with $\sigma\left(t_{f}\right)=\delta>0$. Hence, we can draw the conclusion that $\sigma\left(t_{f}\right)=0$, i.e., $\sigma$ reaches zero at $t=t_{f}$.

Claim 2: Consider the following differential equation,

$$
\dot{\sigma}=-\sigma-\psi(\sigma, t),
$$

where $\psi$ is an RCDF. Then, $\sigma$ reaches zero at $t=t_{f}$.

Proof: We consider a positive initial value $\sigma\left(t_{0}\right)$ for the symmetry of the equation in (14). According to the Comparison Lemma, $\sigma$ is limited by $\phi(t)$ that corresponding to the selected $\psi$ and $t_{a} \leq t_{f}$, as the illustration in Fig. 3(a). Reduction to absurdity is utilized as follows. Let us suppose $t_{a}<t_{f}$.

By overturning the coordinate axis in Fig. 3(a), we have the coordinate axis in Fig. 3(b). The corresponding differential equation in (14) is transformed to:

$$
\frac{\mathrm{d} t}{\mathrm{~d} \sigma}=-\frac{1}{\sigma+\psi} .
$$

We have,

$$
t_{a}=-\int_{\sigma_{0}}^{0} \frac{1}{\sigma+\psi} \mathrm{d} \sigma=\int_{0}^{\sigma_{0}} \frac{1}{\sigma+\psi} \mathrm{d} \sigma .
$$

When $\sigma$ tends to zero, $t$ tends to $t_{a}$, so $t_{f}-t$ will not tend to zero. According to Definition 5, we have $\psi=\lim _{t \rightarrow t_{a}} \frac{\sigma(t)-0}{t_{f}-t} \sim$ $\sigma$. Therefore, $t_{a}=+\infty$, which is conflict with $t_{a}<t_{f}$. The conclusion is drawn that $\sigma$ reaches zero at $t=t_{f}$.
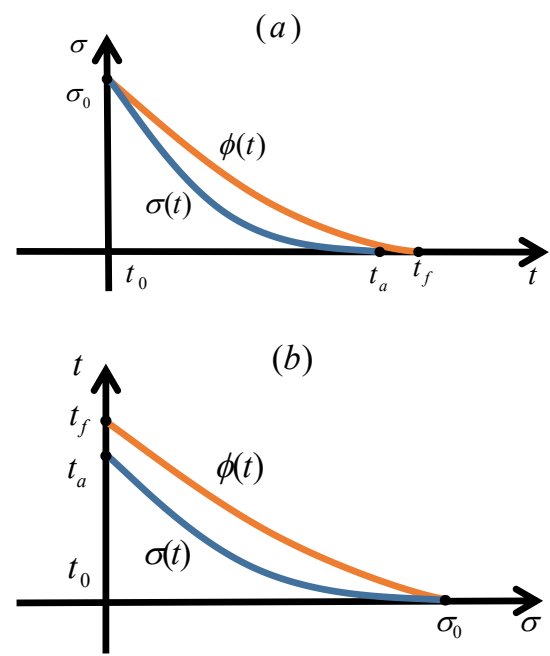

Fig. 3. Abridged general view of the case for Claim 2.

In analogy with Claim 1 and Claim 2, Lemma 1 and Lemma 2 are introduced with the proof in Appendix.

Lemma 1: Consider the following differential equation,

$$
\dot{\sigma}=b(t)-\psi(\sigma, t),
$$

where $\psi$ is an RCDF. If $b(t)$ is bounded but never stabilized at zero until $t=t_{f}$, then $\sigma$ reaches zero at $t=t_{f}$.
Lemma 2: Consider the following differential equation,

$$
\dot{\sigma}=b(t)-\sigma-\psi(\sigma, t),
$$

where $\psi$ is an RCDF. If $b(t)$ is bounded but never stabilized at zero until $t=t_{f}$, then $\sigma$ reaches zero at $t=t_{f}$.

Theorem 1: Consider the system (5) starting from any initial condition of $x$. The controller (11) drives the trajectory of the system (5) to the equilibrium $x=0$ at the selected intant $t=t_{f}$. Moreover, the continuous control $u(t)$ also gradually goes to zero at the instant $t=t_{f}$ and ensures $x(t)=u(t)=$ $0, \forall t \geq t_{f}$. In another word, the equilibrium $x=0$ is PSIS.

Proof: As shown in the last subsection, the controller (11) transforms the system (5) to:

$$
\left\{\begin{array}{l}
\dot{\sigma}_{1}=\sigma_{2}-\psi_{1}, \\
\dot{\sigma}_{i}=\sigma_{i+1}-\sigma_{i}-\psi_{i}, i=2, \ldots, n-1, \\
\dot{\sigma}_{n}=-\sigma_{n}-\psi_{n} .
\end{array}\right.
$$

Consider the last differential equation in (18). According to Claim $2, \sigma_{n}$ monotonously goes to zero at $t_{f}$. Then, $\sigma_{n}$ is taken as $b(t)$, according to Lemma 2, $\sigma_{n-1}$ will go to zero at $t_{f}$. Therefore, $\sigma_{n-2}, \ldots, \sigma_{2}$ can be recursively proved to go to zero at the instant $t_{f}$. Finally, the convergence of $\sigma_{1}$ is a direct result of Lemma 1. Therefore, $\sigma$ is PSIS at $t=t_{f}$ and leads to:

$$
x_{i}\left(t_{f}\right)=\sigma_{i}\left(t_{f}\right)+x_{i, d}\left(t_{f}\right)=x_{i, d}\left(t_{f}\right) .
$$

For the system (5), combining (10) and (11), the relation between the states and the detailed control variables $u_{i}$ is presented as:

$$
\left\{\begin{array}{l}
x_{1}=x_{1, d}=0, \\
x_{2}=x_{2, d}=-\psi_{1}=u_{1}, \\
x_{3}=x_{3, d}=-\dot{\psi}_{1}-\sigma_{2}-\psi_{2}=u_{2}, \\
x_{4}=x_{4, d}=-\ddot{\psi}_{1}-\dot{\sigma}_{2}-\dot{\psi_{2}}-\sigma_{3}-\psi_{3}=u_{3}, \\
\vdots \\
x_{n+1}=x_{n+1, d}=\dot{x}_{n, d}-\sigma_{n}-\psi_{n}=u_{n},
\end{array}\right.
$$

where each equation is formed by $\psi_{i}, \sigma_{i}$, and their derivatives. It is noted that the derivative order of $\psi_{1}$ is the highest among the equations. For $u_{n}$, the derivative order of $\psi_{1}$ is $n-1$ and the derivative order of $\psi_{i}$ is $n-i$. According to Definition 5, when $t$ tends to $t_{f}$, we have $\psi \sim\left(t_{f}-t\right)^{\eta-1}$ and $\psi^{(n-1)} \sim$ $\left(t_{f}-t\right)^{\eta-n}$. As mentioned in Subsection 3.2, for each $\psi_{i}$, the corresponding parameter is selected as $\eta_{i}>n+1-i$, which ensures that the derivative of $\psi_{i}$ tends to zero. Therefore, the proposed control (11) is bounded as $t$ tends to $t_{f}$. Combining the PSIS of $\sigma$, we obtain that the trajectory of the system (5) converges to the equilibrium $x=0$ at the instant $t=t_{f}$. At the same time, $u(t)$ gradually goes to zero and then $u(t)=$ $0, \forall t \geq t_{f}$, which leads to $x(t)=0, \forall t \geq t_{f}$. Hence, the system (5) is PSIS. This completes the proof.

\section{Simulations}

\section{A. Simulations on a Double Integrator System}

Consider a double integrator system,

$$
\left\{\begin{array}{l}
\dot{x}_{1}=x_{2} \\
\dot{x}_{2}=u
\end{array}\right.
$$


According to (10) and (11), the detailed controller is:

$$
u_{2}= \begin{cases}-\frac{\partial \psi_{1}}{\partial x_{1}}\left(\sigma_{2}-\psi_{1}\right)-\frac{\partial \psi_{1}}{\partial t} & \\ -\sigma_{2}-\psi_{2}, & t_{0} \leq t<t_{f}, \\ 0, & t_{f} \leq t .\end{cases}
$$

The detailed RCDFs are selected as:

$$
\left\{\begin{array}{l}
\psi_{1}=\frac{\eta_{1} \sigma_{1}}{t_{f}-t}, \eta_{1}=3 \\
\psi_{2}=\frac{\eta_{2} \sigma_{2}}{t_{f}-t}, \eta_{2}=2
\end{array}\right.
$$

In simulations, we choose $t_{f}=1 \mathrm{~s}$ and three different sets of initial values: set 1: $x_{1}(0)=1, x_{2}(0)=-3$; set $2: x_{1}(0)=$ $2, x_{2}(0)=-1$; set $3: x_{1}(0)=3, x_{2}(0)=1$. The simulation results are depicted as Fig. 4. Fig. 4(a) shows the trajectories of $x_{1}$, Fig. 4(b) shows the trajectories of $x_{2}$, Fig. 4(c) shows the trajectories of control variables under the three different initial conditions.

(a)

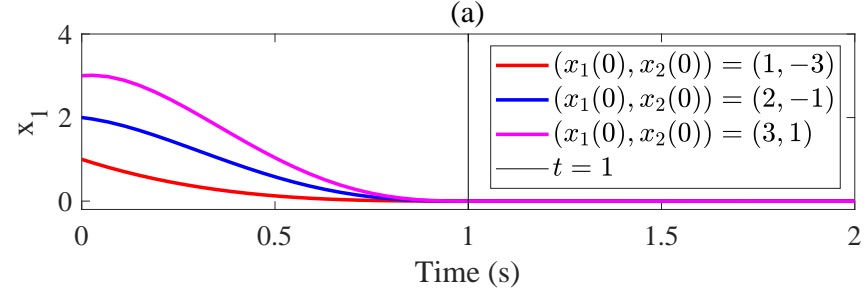

(b)

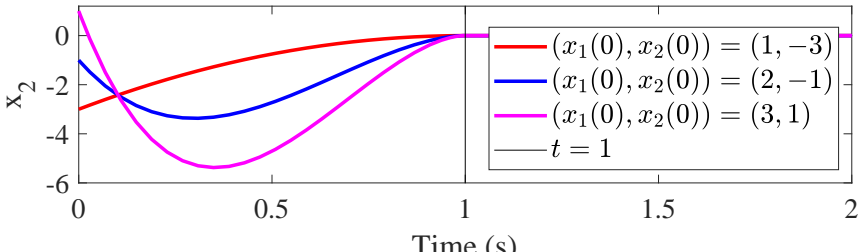

(c)

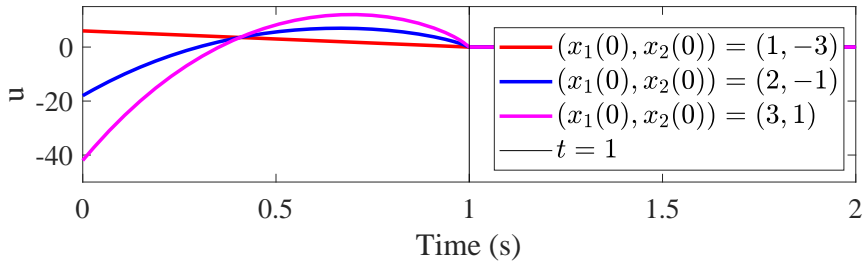

Fig. 4. States and control variables of the double integrator system.

\section{B. Simulations on a Simple Pendulum System}

Consider a simple pendulum system [32]. $l=0.5 \mathrm{~m}$ is the length of the rod and $m=0.1 \mathrm{~kg}$ denotes the mass of the bob. The rod is assumed to be rigid and has zero mass. The acceleration due to gravity is $\mathrm{g}=9.81 \mathrm{~m} / \mathrm{s}^{2}$ and the friction coefficient is denoted as $k=0.01$. Let $\theta(\mathrm{rad})$ denote the angle subtended by the rod and the vertical axis through the pivot point. Applying a torque $T$ to the pendulum, we have,

$$
m l \ddot{\theta}=-m g \sin \theta-k l \dot{\theta}+\frac{1}{l} T .
$$

Taking the states as $x_{1}=\theta=y$ and $x_{2}=\dot{\theta}$, we have,

$$
\left\{\begin{array}{l}
\dot{x}_{1}=x_{2} \\
\dot{x}_{2}=-\frac{g}{l} \sin x_{1}-\frac{k}{m} x_{2}+\frac{1}{m l^{2}} T .
\end{array}\right.
$$

Let the torque $T$ be

$$
T=m l^{2}\left(\frac{g}{l} \sin x_{1}+\frac{k}{m} x_{2}+u\right) .
$$

We have,

$$
\left\{\begin{array}{l}
\dot{x}_{1}=x_{2}, \\
\dot{x}_{2}=u
\end{array}\right.
$$

Assume the initial value $x_{1}(0)=0.09 \mathrm{rad}$ and $x_{2}(0)=$ $0.1 \mathrm{rad} / \mathrm{s}$. Our goal is to make $x_{1}=0.15 \mathrm{rad}$ at $t_{f}=0.5 \mathrm{~s}$, i.e., $x_{1, d}=0.15 \mathrm{rad}$. We replace the variable $x_{1}$ as $\sigma_{1}=x_{1}-x_{1, d}$ in the controller presented in (20) and then obtain

$$
u_{2}= \begin{cases}-\frac{\partial \psi_{1}}{\partial x_{1}}\left(\sigma_{2}-\psi_{1}\right)-\frac{\partial \psi_{1}}{\partial t}-\sigma_{2}-\psi_{2}, & t_{0} \leq t<t_{f} \\ 0, & t_{f} \leq t .\end{cases}
$$

The detailed RCDFs are also selected as:

$$
\left\{\begin{array}{l}
\psi_{1}=\frac{\eta_{1} \sigma_{1}}{t_{f}-t}, \eta_{1}=3, \\
\psi_{2}=\frac{\eta_{2}\left(\sigma_{2}^{2}+1\right) \arctan \left(\sigma_{2}\right)}{t_{f}-t}, \eta_{2}=2 .
\end{array}\right.
$$

The simulation result is depicted as Fig. 5. Fig. 5(a) shows the states of the pendulum system and Fig. 5(b) shows the control variables.

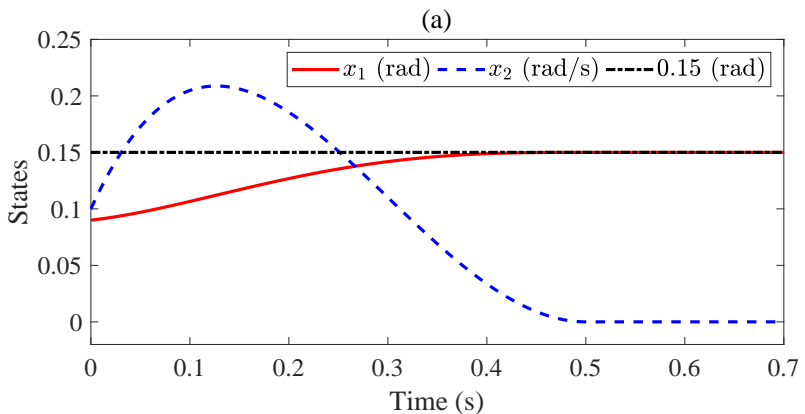

(b)

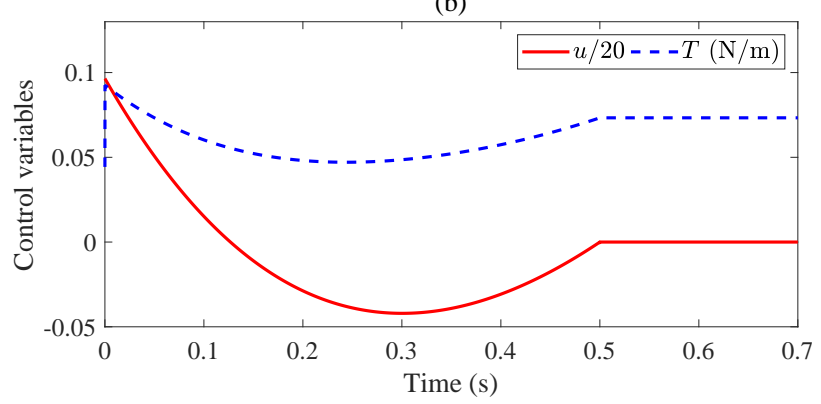

Fig. 5. States and control variables of the pendulum system.

\section{Simulations on a Magnetic Suspension}

Consider the magnetic suspension [32]. The vertical (downward) position of the ball measured from a reference point is denoted as $z_{1}$, which is also the output $y . z_{2}$ denotes the speed of the ball and $z_{3}$ denotes the current of the electromagnet. The state equations are:

$$
\left\{\begin{array}{l}
\dot{z}_{1}=z_{2} \\
\dot{z}_{2}=g-\frac{k}{m} z_{2}-\frac{L_{0} a z_{3}^{2}}{2 m\left(a+z_{1}\right)^{2}} \\
\dot{z}_{3}=g-\frac{1}{L_{1}+\frac{a L_{0}}{a+z_{1}}}\left[-R z_{3}+\frac{L_{0} a z_{2} z_{3}}{\left(a+z_{1}\right)^{2}}+v\right]
\end{array}\right.
$$


where the voltage $v$ is the control input. We assume $m=$ $0.1 \mathrm{~kg}, k=0.001 \mathrm{~N} / \mathrm{m} / \mathrm{s}, g=9.81 \mathrm{~m} / \mathrm{s}^{2}, a=0.05 \mathrm{~m}, L_{0}=$ $0.01 \mathrm{H}, L_{1}=0.02 \mathrm{H}$, and $R=1 \Omega$. By choosing

$$
\left\{\begin{array}{l}
x_{1}=y=z_{1}, \\
x_{2}=\dot{y}=z_{2}, \\
x_{3}=\ddot{y}=g-\frac{k}{m} z_{2}-\frac{L_{0} a z_{3}^{2}}{2 m\left(a+z_{1}\right)^{2}},
\end{array}\right.
$$

one can obtain:

$\left\{\begin{array}{l}\dot{x}_{1}=x_{2}, \dot{x}_{2}=x_{3}, \\ \dot{x}_{3}=-\frac{k}{m} x_{3}-\frac{a L_{0} z_{3}}{m\left(a+z_{1}\right)^{2}\left(L_{1}+\frac{a L_{0}}{a+z_{1}}\right)}\left(-R z_{3}-\frac{L_{1} z_{2} z_{3}}{a+z_{1}}+v\right) .\end{array}\right.$

As shown in Subsection 2.2, let the control be:

$$
v=R z_{3}+\frac{L_{1} z_{2} z_{3}}{a+z_{1}}-\frac{m\left(a+z_{1}\right)^{2}\left(L_{1}+\frac{a L_{0}}{a+z_{1}}\right)}{a L_{0} z_{3}}\left(\frac{k}{m} x_{3}+u\right) .
$$

Then, we obtain that

$$
\left\{\begin{array}{l}
\dot{x}_{1}=x_{2}, \\
\dot{x}_{2}=x_{3}, \\
\dot{x}_{3}=u .
\end{array}\right.
$$

Assume the initial value $x_{1}(0)=z_{1}(0)=0.09 \mathrm{~m}, x_{2}(0)=$ $z_{2}(0)=-0.03 \mathrm{~m} / \mathrm{s}$ and $z_{3}(0)=-6.6 \mathrm{~A}$. Our goal is to stabilize $x_{1}=z_{1}=0.05 \mathrm{~m}$ at $t_{f}=0.5 \mathrm{~s}$, i.e., $x_{1, d}=0.05 \mathrm{~m}$. We replace the variable $x_{1}$ by $\sigma_{1}=x_{1}-x_{1, d}$ in the controller presented in (10) and (11) and then obtain

$$
u_{3}=\left\{\begin{array}{cl}
-\sigma_{3}-\psi_{3}-\frac{\partial x_{3, d}}{\partial x_{1}}\left(\sigma_{2}-\psi_{1}\right)-\frac{\partial x_{3, d}}{\partial t} & \\
-\frac{\partial x_{3, d}}{\partial \sigma_{2}}\left(\sigma_{3}-\sigma_{2}-\psi_{2}\right), & t_{0} \leq t<t_{f}, \\
0, & t_{f} \leq t .
\end{array}\right.
$$

Specifically, $\psi_{i}$ is chosen as:

$$
\left\{\begin{array}{l}
\psi_{1}=-\frac{\eta_{1} \sigma_{1}}{t_{f}-t}, \eta_{1}=5 \\
\psi_{2}=-\frac{\eta_{2}\left(1-\mathrm{e}^{-\left|\sigma_{2}\right|}\right)}{t_{f}-t} \operatorname{sign}\left(\sigma_{2}\right), \eta_{2}=4 \\
\psi_{3}=-\frac{\eta_{3}\left(\sigma_{3}^{2}+1\right) \arctan \left(\sigma_{3}\right)}{t_{f}-t}, \eta_{3}=3
\end{array}\right.
$$

Fig. 6(a) shows the states of the magnetic suspension system and Fig. 6(b) shows the control variables. The initial value of the real controller $v$ is about $-7 \mathrm{~V}$. Moreover, $u$ is bounded and goes to zero exactly at $t_{f}=0.5$.

Remark 7: Although the convergence time can be arbitrarily chosen, the magnitude of the control will inevitably be very large when the values of the initial condition are very large or the regulation time is set to be very small. This is unexpected in practice [19]. Hence, the bound of the control variables will be considered qualitatively in our future study.

\section{CONCLUSIONS}

This paper has dealt with the problem to stabilize system states to the origin at a selected time instant irrespective of initial system states and parameters. This can be achieved by the feature of a series of well-designed time-varying feedback functions, which are easy to be obtained according to the presented guidance and are defined as RCDFs. We directly considered the feature of differential equations instead of the Lyapunov method, and then reduction to absurdity has been applied and the proof is unexpectedly elegant. In addition to (a)

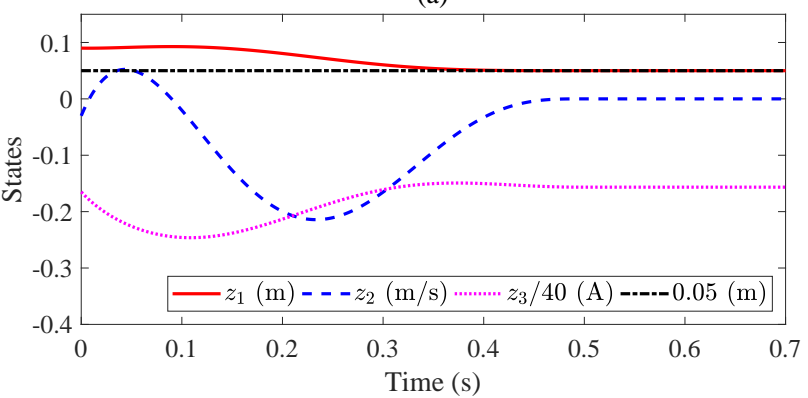

(b)

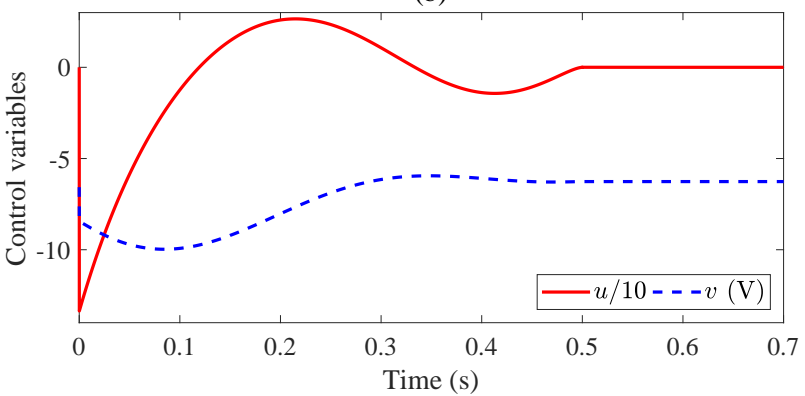

Fig. 6. States and control of the magnetic suspension system.

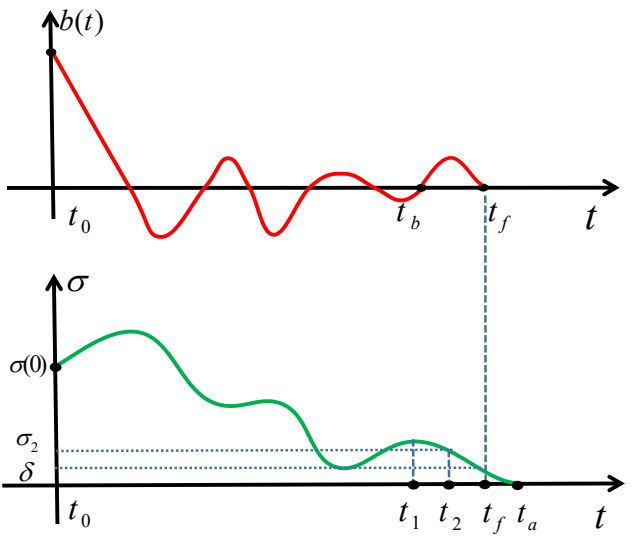

Fig. 7. The sketch map of Case 1 for Lemma 1.

mathematical proof, the control scheme has also been verified by numerical simulations. This control strategy would be of great importance in industrial and engineering applications with the requirement of the precise time.

\section{APPENDIX}

Proof of Lemma 1: Set $t_{b}$ the last time when $b(t)$ passed through zero ( $t_{b}=t_{0}$ if $b(t)$ never passes through zero). $b(t)$ may have same or opposite sign with $\sigma\left(t_{b}\right)$. We consider a positive initial value $\sigma\left(t_{b}\right)$. Then, $b(t)$ with $t \in\left(t_{b}, t_{f}\right)$ could be cased by positive or negative. It is obvious that $\sigma$ will converge to zero at $t_{f}$ if $\dot{\sigma}=-\psi(\sigma, t)$. Now, let us consider:

$$
\dot{\sigma}=b(t)-\psi(\sigma, t) \text {. }
$$

Case 1. $b(t)>0, t \in\left(t_{b}, t_{f}\right)$, of which an illustration is shown in Fig. 7. According to the Comparison Lemma, the real convergence time of the equation in (21) would be $t_{a} \geq t_{f}$. Reduction to absurdity is utilized as follows. 


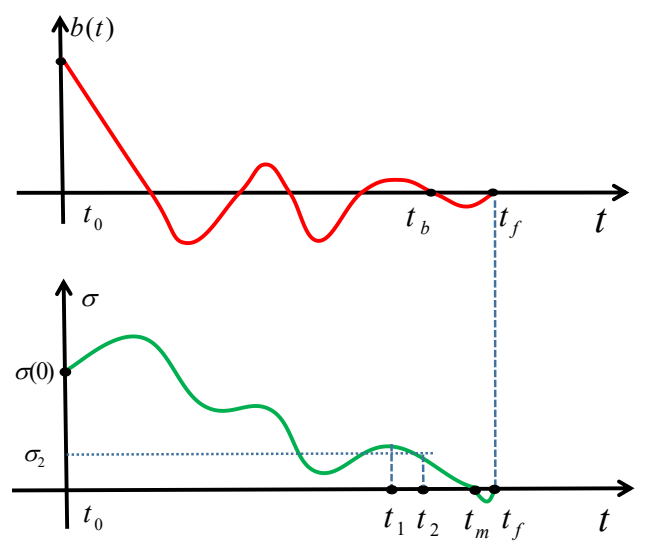

Fig. 8. The sketch map of Case 2 for Lemma 2.

Assume that $t_{a}>t_{f}$, i.e., $\sigma\left(t_{f}\right)=\delta>0$. Based on the form of $\psi$ and the fact that $b(t)$ is bounded, $\exists t_{1}$, s.t. $\sigma^{\prime}(t)<$ $0, t \in\left(t_{1}, t_{f}\right)$. Taking $t_{2} \geq \max \left(t_{1}, t_{b}\right), \sigma\left(t_{2}\right)=\sigma_{2}$, we have,

$$
\sigma\left(t_{f}\right)=\sigma_{2}+\int_{t_{2}}^{t_{f}} b(t) \mathrm{d} t-\int_{t_{2}}^{t_{f}} \frac{\zeta(\sigma)}{t_{f}-t} \mathrm{~d} t
$$

where $\frac{\zeta(\sigma)}{t_{f}-t}$ is $\psi$. Utilizing the mean value theorem of integrals, we have,

$$
\sigma\left(t_{f}\right)=k-\int_{t_{2}}^{t_{f}} \frac{\zeta(\sigma)}{t_{f}-t} \mathrm{~d} t
$$

where $k$ is a constant. As shown in the proof of Claim 1, one can obtain $\sigma\left(t_{f}\right)=-\infty$, which is conflicted with the assumption that $\sigma\left(t_{f}\right)=\delta>0$. Therefore, $\sigma\left(t_{f}\right)=0, t_{a}=$ $t_{f}$.

Case 2. $b(t)<0, t \in\left(t_{b}, t_{f}\right)$, of which an illustration is shown in Fig. 8. There exists three subcases:

(i) The value of $b(t)$ cannot drive $\sigma$ to be negative or zero before $t_{f}$. According to the sign of $b(t), \sigma$ will tend to zero at or before the instant $t_{f}$ (due to the Comparison Lemma). So, $\sigma$ reaches zero at the instant $t_{f}$.

(ii) The value of $|b(t)|$ is large enough and drive $\sigma$ to be zero at $t_{m} \in\left(\max \left(t_{1}, t_{b}\right), t_{f}\right)$. Because $b(t)<0, t \in\left(t_{b}, t_{f}\right)$, $\sigma$ will go across zero and it turns to the subcase (iii).

(iii) The value of $|b(t)|$ is large enough and drive $\sigma$ to be negative at $t_{m} \in\left(\max \left(t_{1}, t_{b}\right), t_{f}\right)$. Then $b(t)$ and $\sigma$ have the same sign and the proof on $\left(t_{m}, t_{f}\right)$ is similar to Case 1.

Combining Case 1 and Case 2, we know that $\sigma$ will reach zero at the instant $t_{f}$.

Proof of Lemma 2: The proof imitates that of Lemma 1 and the differential equation (14) is taken as a reference differential equation to utilize the Comparison Lemma. The only difference is that (22) is replaced by

$$
\sigma\left(t_{f}\right)=\sigma_{2}+\int_{t_{2}}^{t_{f}} b(t) \mathrm{d} t-\int_{t_{2}}^{t_{f}} \sigma \mathrm{d} t-\int_{t_{2}}^{t_{f}} \frac{\zeta(\sigma)}{t_{f}-t} \mathrm{~d} t .
$$

Then, the detailed proof can be easily completed by following that of Lemma 1 and is thus omitted.

\section{REFERENCES}

[1] Y. Orlov, "Finite time stability and robust control synthesis of uncertain switched systems," SIAM Journal on Control and Optimization, vol. 43, no. 4, pp. 1253-1271, 2004.
[2] Z. Y. Sun, M. M. Yun, and T. Li, "A new approach to fast global finitetime stabilization of high-order nonlinear system," Automatica, vol. 81, pp. 455-463, 2017.

[3] S. P. Bhat and D. S. Bernstein, "Finite-time stability of continuous autonomous systems," SIAM Journal on Control and Optimization, vol. 38, no. 3, pp. 751-766, 2000.

[4] _ - "Geometric homogeneity with applications to finite-time stability," Mathematics of Control Signals and Systems, vol. 17, no. 2, pp. 101127, 2005.

[5] A. Polyakov, D. Efimov, and W. Perruquetti, "Finite-time and fixed-time stabilization: Implicit lyapunov function approach," Automatica, vol. 51, pp. 332-340, 2015.

[6] E. Cruz-Zavala, J. A. Moreno, and L. M. Fridman, "Fast finite-time stability and its application in adaptive control of high-order nonlinear system," Automatica, vol. 106, pp. 339-348, 2019.

[7] — "Uniform robust exact differentiator," IEEE Transactions on Automatic Control, vol. 56, no. 11, pp. 2727-2733, 2011.

[8] A. Polyakov, "Nonlinear feedback design for fixed-time stabilization of linear control systems," IEEE Transactions on Automatic Control, vol. 57, no. 8, pp. 2106-2110, 2012.

[9] M. T. Angulo, J. A. Moreno, and L. M. Fridman, "Robust exact uniformly convergent arbitrary order differentiator," Automatica, vol. 49 , pp. 2489-2495, 2013.

[10] T. Menard, E. Moulay, and W. Perruquetti, "Fixed-time observer with simple gains for uncertain systems," Automatica, vol. 81, pp. 438-446, 2017.

[11] A. Polyakov, D. Efimov, and W. Perruquetti, "Robust stabilization of mimo systems in finite/fixed time," International Journal of Robust and Nonlinear Control, vol. 26, no. 1, pp. 69-90, 2016.

[12] J. D. Sanchez-Torres, E. N. Sanchez, and A. G. Loukianov, "A discontinuous recurrent neural network with predefined time convergence for solution of linear programming," in Swarm Intelligence, 2014.

[13] — , "Predefined-time stability of dynamical systems with sliding modes," in American Control Conference (ACC), 2015.

[14] J. D. Sanchez-Torres, D. Gmez-Gutirrez, E. Lopez, and A. G. Loukianov, "A class of predefined-time stable dynamical systems," IMA Journal of Mathematical Control and Information, vol. 35, no. Supplement 1, pp. i1-i29, 2017.

[15] E. Jimenez-Rodrguez, A. Muoz-Vzquez, J. D. Sanchez-Torres, and A. G. Loukianov, "A note on predefined-time stability," IFAC-PapersOnLine, vol. 51, no. 13, pp. 520-525, 2018.

[16] S. Seo, H. Shim, and H. S. Jin, "Global finite-time stabilization of a nonlinear system using dynamic exponent scaling," in IEEE Conference on Decision and Control, 2008.

[17] Y. Song, Y. Wang, J. Holloway, and M. Krstic, "Time-varying feedback for regulation of normal-form nonlinear systems in prescribed finite time," Automatica, vol. 83, pp. 243-251, 2017.

[18] Y. Song, Y. Wang, and M. Krstic, "Time-varying feedback for stabilization in prescribed finite time," International Journal of Robust and Nonlinear Control, vol. 29, no. 2, pp. 618-633, 2018.

[19] B. Zhou, "Finite-time stabilization of linear systems by bounded linear time-varying feedback," Automatica, vol. 113, p. 108760, 2020.

[20] Y. Chitour, R. Ushirobira, and H. Bouhemou, "Stabilization for a perturbed chain of integrators in prescribed time," SIAM Journal on Control and Optimization, vol. 58, no. 2, pp. 1022-1048, 2020.

[21] P. Krishnamurthy, F. Khorrami, and M. Krstic, "A dynamic high-gain design for prescribed-time regulation of nonlinear systems," Automatica, vol. 115 , p. 108860, 2020.

[22] X. D. Chen, X. F. Zhang, and Q. R. Liu, "Prescribed-time decentralized regulation of uncertain nonlinear multi-agent systems via output feedback," Systems and Control Letters, vol. 137, p. 104640, 2020.

[23] Y. Wang, Y. Song, D. J. Hill, and M. Krstic, "Prescribed-time consensus and containment control of networked multiagent systems," IEEE Transactions on Cybernetics, vol. 49, no. 4, pp. 1138-1147, 2019.

[24] D. Gomez-Gutierrez, "On the design of non-autonomous fixed-time controllers with a predefined upper bound of the settling time," International Journal of Robust and Nonlinear Control, vol. 30, no. 10, pp. 38713885, 2020.

[25] W. Michiels and B. Zhou, "On the fixed-time stabilization of input delay systems using act-and-wait control," Systems and Control Letters, vol. 146, p. 104807, 2020.

[26] C. Hu, H. B. He, and H. J. Jiang, "Fixed/preassigned-time synchronization of complex networks via improving fixed-time stability," IEEE Transactions on Cybernetics, vol. 51, no. 6, pp. 2882-2892, 2021.

[27] H. G. Zhang, J. Duan, Y. C. Wang, and Z. Y. Gao, "Bipartite fixed-time output consensus of heterogeneous linear multiagent systems," IEEE Transactions on Cybernetics, vol. 51, no. 2, pp. 548-557, 2021. 
[28] G. Kunal and P. Dimitra, "Fixed-time stable gradient flows: Applications to continuous-time optimization," IEEE Transactions on Automatic Control, vol. 66, no. 5, pp. 2002-2015, 2021.

[29] O. Mechali, L. Xu, Y. Huang, M. Shi, and X. Xie, "Observer-based fixedtime continuous nonsingular terminal sliding mode control of quadrotor aircraft under uncertainties and disturbances for robust trajectory tracking: Theory and experiment," Control Engineering Practice, vol. 111, p. 104806, 2021.

[30] J. Cortes, "Discontinuous dynamical systems," IEEE Control Systems Magazine, vol. 28, no. 3, pp. 36-73, 2008.

[31] E. Jimenez-Rodriguez, A. J. Muoz-Vazquez, J. D. Sanchez-Torres, M. Defoort, and A. G. Loukianov, "A Lyapunov-like characterization of predefined-time stability," IEEE Transactions on Automatic Control, vol. 65, no. 11, pp. 4922-4927, 2019.

[32] H. K. Khalil, Nonlinear systems (3rd ed.). Prentice-Hal, 2002.

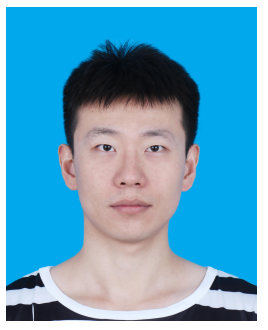

Kuang Jiyuan received the B.M. degree in control science and engineering in 2016, the M.E. degree in power electronics and power drives in 2019, both from Shandong University, Jinan, China. He is currently working toward the Ph.D. degree in Control Science and Engineering from Harbin Institute of Technology, Harbin, China. His current research interests include prescribed time stability, sliding mode control and power electronics systems.

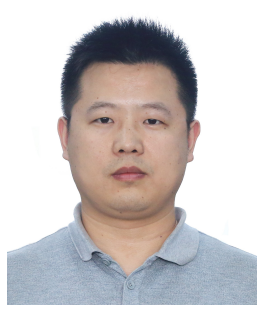

Jianxing Liu (M13-SM20) received the B.S. degree in mechanical engineering and the M.E. degree in control science and engineering from the Harbin Institute of Technology, Harbin, China, in 2004 and 2010 , respectively, and the Ph.D. degree in automation from the Technical University of Belfort- Montbeliard, Belfort, France, in 2014. Since 2014, he has been with the Harbin Institute of Technology. He is currently a Professor in the Department of Control Science and Engineering. His current research interests include sliding mode control, nonlinear control and observation, industrial electronics, and renewable energy solutions. He is involved in the Data Driven Control and Monitoring Technical Committee of the IEEE Industrial Electronics Society and is currently serving as an Associate Editor of the ISA Transactions and IEEE Journal of Emerging and Selected Topics in Industrial Electronics.

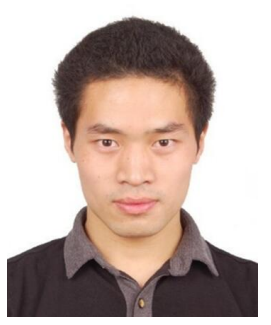

Yabin Gao (M'19) received the B.M. degree in Information Management and Information System in 2012, the M.E. degree in Software Engineering in 2015, both from Bohai University, Jinzhou, China, and the Ph.D. degree in Control Science and Engineering from Harbin Institute of Technology, Harbin, China, in 2020. He is currently a lecturer in the Department of Control Science and Engineering, Harbin Institute of Technology. From October 2017 to October 2019, he was a visiting scholar in the Department of Mechanical Engineering, University of Victoria, Victoria, Canada.

His current research interests include sliding mode control, intelligent control, robust filtering, fault detection, cyber-physical systems, and their applications.

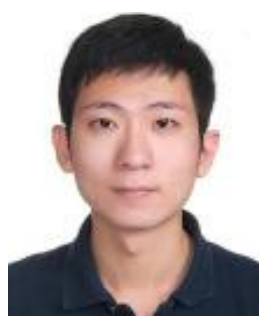

Chih-Chiang Chen received the Ph.D. degree in control theory from National Chiao Tung University, Hsinchu, Taiwan, in 2017. From 2015 to 2016, he was a visiting scholar at the Department of Electrical and Computer Engineering, University of Texas at San Antonio, USA. Since August 2017, he has been with the Department of Systems and Naval Mechatronic Engineering, National Cheng Kung University, Taiwan, where he is currently an assistant professor. His current research interests include nonsystem theory. linear control, dynamic systems, and homogeneous

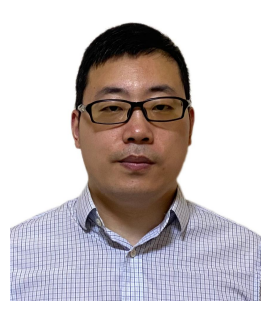

Xiaoju Zhang received the B.M. degree in Mathematics and Applied Mathematics in 2011 from Zhengzhou University, Zhengzhou, China; the M.E. degree in Applied Mathematics in 2014 from University of Chinese Academy of Sciences, Beijing, China. He is currently working toward the Ph.D. degree in Mathematics from Harbin Institute of Technology, Harbin, China. His current research interests include existence, uniqueness, asymptotic behavior and blow-up of solutions for nonlinear fractional partial differential equations.

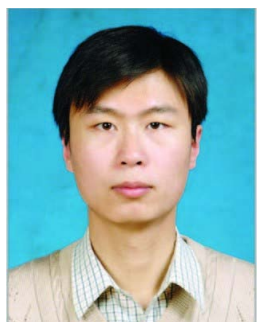

Guanghui Sun received the B.S. degree in automation and the M.S. and Ph.D. degrees in control science and engineering from Harbin Institute of Technology, Harbin, China, in 2005, 2007, and 2010, respectively. He is currently a Professor in the Department of Control Science and Engineering, Harbin Institute of Technology. His research interests include fractional-order systems, networked control systems, and sliding mode control. 